\title{
Evaluation of the Expression of the Human Epithelial Receptor 2 (HER2) in Gastric Carcinoma
}

\author{
Roberto de Moraes Cordts Filho, Paulo Kassab, Laura Carolina Lopez Claro, \\ Mabel Tatty de Medeiros Fracassi, Patrícia Colombo-Souza, Daniel Kenji Fukuhara, \\ Fábio Rodrigues Thuler, Wilson Rodrigues de Freitas Junior, \\ Elias Jirjoss Ilias, and Carlos Alberto Malheiros
}

Gastric and Obesity Surgery Division, Surgery Department, Santa Casa of São Paulo Medical School, São Paulo, SP, Brazil

Correspondence should be addressed to Roberto de Moraes Cordts Filho; bobcordts@terra.com.br

Received 3 May 2016; Accepted 6 December 2016

Academic Editor: ChangHui Shen

Copyright (C) 2016 Roberto de Moraes Cordts Filho et al. This is an open access article distributed under the Creative Commons Attribution License, which permits unrestricted use, distribution, and reproduction in any medium, provided the original work is properly cited.

\begin{abstract}
Objective. To evaluate the HER2 expression on gastric adenocarcinoma from a Brazilian population and also to analyze the relations between the receptor and clinical characteristics, as well as the survival status. Materials and Methods. A retrospective analysis was conducted from January of 2008 to July of 2012, considering only gastrectomies with curative intent. Tumors were tested for HER2 status using immunohistochemistry. The relation between HER2 status and clinical aspects, surgical findings, and survival were also analyzed. Results. 222 patients with gastric carcinoma were submitted to surgery during that period, but only $121(54,5 \%)$ were with curative intention. The immunohistochemistry revealed that 4 patients $(3,3 \%)$ were HER2-positive, 6 patients $(4,9 \%)$ HER2undetermined, and 111 patients (91,7\%) HER2-negative. There was no statistical concordance between HER2 status and survival or the clinical aspects. Conclusion. The HER2 overexpression rate was very low in this Brazilian population sample and cannot be considered as a prognostic factor.
\end{abstract}

\section{Introduction}

Gastric carcinoma is one of the most common tumors in the world. An estimate made by the National Cancer Institute of the Brazilian Health Ministry (INCA) for 2016 was 12,920 new cases in men and 7,600 in women, occupying the fourth position for men and fifth for women [1]; in the world setting, it is found with a frequency which varies from second to fifth position, depending on the region under evaluation, with more than $70 \%$ of the cases occurring in developing countries [2]. Even though much has been accomplished in the combat of this disease, it still persists as one of the most aggressive tumors; its survival rate of 5 years is approximately $60 \%$, and this drops to 2 to $5 \%$ once metastases have been identified. In this manner, the molecular aspects have attracted even more attention on the part of researchers as a promising therapeutic approach, especially in more advanced cases whose surgical limitations reduce the treatment options even further.
The epithelial growth factor receptors (EGFR: HER1, HER2, HER3, and HER4) are cellular membrane structures expressed in several healthy tissues and in various tumors, particularly in those of epithelial origin, and their activation apparently has an important relationship with the genesis of these tumors, due to the stimulation of cellular proliferation stimulus and the inhibition of apoptosis [3]. The superexpression of HER2 has been closely related to breast cancer, colorectal carcinoma, and nonsmall cell lung cancer, in fact suggesting a worse prognosis $[4,5]$. In esophagogastric carcinoma, the expression appears to vary between 5 and $25 \%$ $[6,7]$, without reliable data as to its prognosis [8-11].

Over the last few years, diverse medications having an effect on EGFR have been developed. One specific group, which has presented promising results [12-14], is that of the monoclonal antibodies (cetuximab and trastuzumab) that bond with the extracellular domain of the receptor. Another group of agents includes tyrosine-kinase inhibitors 
(gefitinib, lapatinib, and erlotinib), acting on the intracellular level, which may present good results when associated with conventional chemotherapeutic treatments [15-17].

The primary objective of this study was to evaluate the positivity of the HER2 expression in gastric adenocarcinomas in the Brazilian population that had undergone surgery. Secondly, we investigated the relationship between this receptor and the patient characteristics and their possible influence on the prognosis.

\section{Materials and Methods}

2.1. Patients. A retrospective analysis was made based on prospectively acquired data, collected from a specific protocol filled in every gastrectomy due to gastric adenocarcinoma performed with curative intent at the Hospital of Santa Casa of São Paulo Medical School from January 2008 to July 2012. All were submitted to surgery with curative potential (free margins, negative cytology, and adequate lymphadenectomy), independent of the stage, histological type or any other variable. The follow-up time was at least 3 years or until death. Perioperative deaths were excluded.

Right after the resection, the specimens were fixed in 10\% neutral buffered formalin for a period not less than $18 \mathrm{~h}$ or more than $24 \mathrm{~h}$, as recommended by the Pathology Department of our institution, as well as the reagents' manufacturer. The tissues then were sliced and conserved in paraffin. The paraffin blocks that presented the best tumor representation were analyzed on slides stained with hematoxylin-eosin. Once the block had been chosen, the material was sliced into new sections measuring 3 to $5 \mu \mathrm{m}$ on a microtome and placed on silanized slides. The whole process of obtaining the immunohistochemical reaction was performed with the HercepTest ${ }^{\circledR}$, using the machine AutoStainer Plus ${ }^{\circledR}$, following the manufacturer's instructions (both $D a k o^{\circledR}$ ).

The processes of deparaffinization and hydration were performed at room temperature in xylene and alcohol baths, finishing in a distilled water bath. Antigenic recovery was then performed making use of a solution supplied in the kit and an endogenous peroxidase blocker (incubation in a humid chamber for 5 minutes with $100 \mu \mathrm{L}$ of the solution on each slide). For all of the processes, $300 \mu \mathrm{L}$ of the primary antibody anti-HER-2/neu, a polyclonal developed by Dako (code A0485) from rabbits, was applied to each slide. The secondary antibody supplied in the kit, called visualizing reagent, is a dextran polymer conjugated from horseradish with anti-rabbit goat immunoglobulins. A quantity of $100 \mu \mathrm{L}$ of this reagent was used on each slide. Lastly, $100 \mu \mathrm{L}$ of the $5 \%$ chromogen diaminobenzidine (DAB) was placed on each slide and subsequently washed with distilled water, which gave the slides the typical brown color of the reaction. The counter-staining was performed with hematoxylin to identify the nuclei, in order not to have doubts as to the identification and reading of the membrane coloring. The slides were then washed, dehydrated, and assembled. The controls used were obtained from breast adenocarcinoma specimens with a known strong positive reaction (score $3+$ ) to HER2.

All of the slides, both hematoxylin-eosin and immunohistochemical, were evaluated by a very capable pathologist
(Dr. L. Claro). Following well-established protocols [18], the interpretation of the slides was based on the positivity in brown on the cellular membrane, quantified and graded according to the information on the antibody insert.

(i) Score 0: absence of color or reaction in less than $10 \%$ of neoplastic cells; interpretation = negative;

(ii) Score 1: weak and/or incomplete coloring of the membrane in more than $10 \%$ of the neoplastic cells; interpretation $=$ positive $1+$;

(iii) Score 2: moderate and/or incomplete coloring of membrane in more than $10 \%$ of the neoplastic cells; interpretation $=$ positive $2+$;

(iv) Score 3: strong and complete and/or incomplete of the membrane in more than $10 \%$ of the neoplastic cells; interpretation $=$ positive $3+$.

Following what is already widely accepted in the literature, we stratified the results obtained in this manner: score 0 and 1+ defined as absent HER2; score 2+ defined as indeterminate HER2; and score 3+ considered positive HER2.

There was no follow-up loss and the histopathological results were analyzed by both the author and the same pathologist. The adopted classification for the tumor invasion $(\mathrm{T})$, lymph node involvement $(\mathrm{N})$, and final staging was that of the Japanese Gastric Cancer Association, 3rd English Edition [19]. There was no sponsorship in the acquisition of the kits.

2.2. Statistical Analysis. In order to determine the association between the presence, indetermination, or absence of the HER2 protein and the studied characteristics, the Chi-square or Fisher's exact tests were used, fixing the rejection level of the null hypothesis at 0.05 or $5 \%$, or in other words, with $p<$ 0.05 being considered significant. To analyze the difference in ages (years), survival time (months), and number of positive lymph nodes among the presence, indetermination, and absence of the HER2 protein, the Kruskal-Wallis variance analysis was utilized, also fixing at 0.05 , or $5 \%$, the rejection level of the null hypothesis. The software used for the statistical analysis was the Statistical Package for the Social Sciences (SPSS) version 18.0 and the Bioestast version 5.3.

2.3. Ethical Aspects. This study was analyzed and approved by the Ethics Committee of the Education and Research Institute at the Santa Casa of São Paulo Medical School (registry number 44942013.0.00005479). There was no sponsorship whatsoever by any medical companies and the whole of the study followed the most rigid protocols for research using human beings (Helsinki Declaration).

\section{Results}

During the period of January 2008 to July 2012, 222 patients with gastric adenocarcinoma were treated by the Stomach and Obesity Surgery Department of Santa Casa of São Paulo Medical School. However, only 121 (54.5\%) were submitted to surgery with a curative potential and thus being considered eligible for the study. Tables 1 and 2 present the patient 
TABLE 1: Characteristics of patients and their relationship with the expression of HER2.

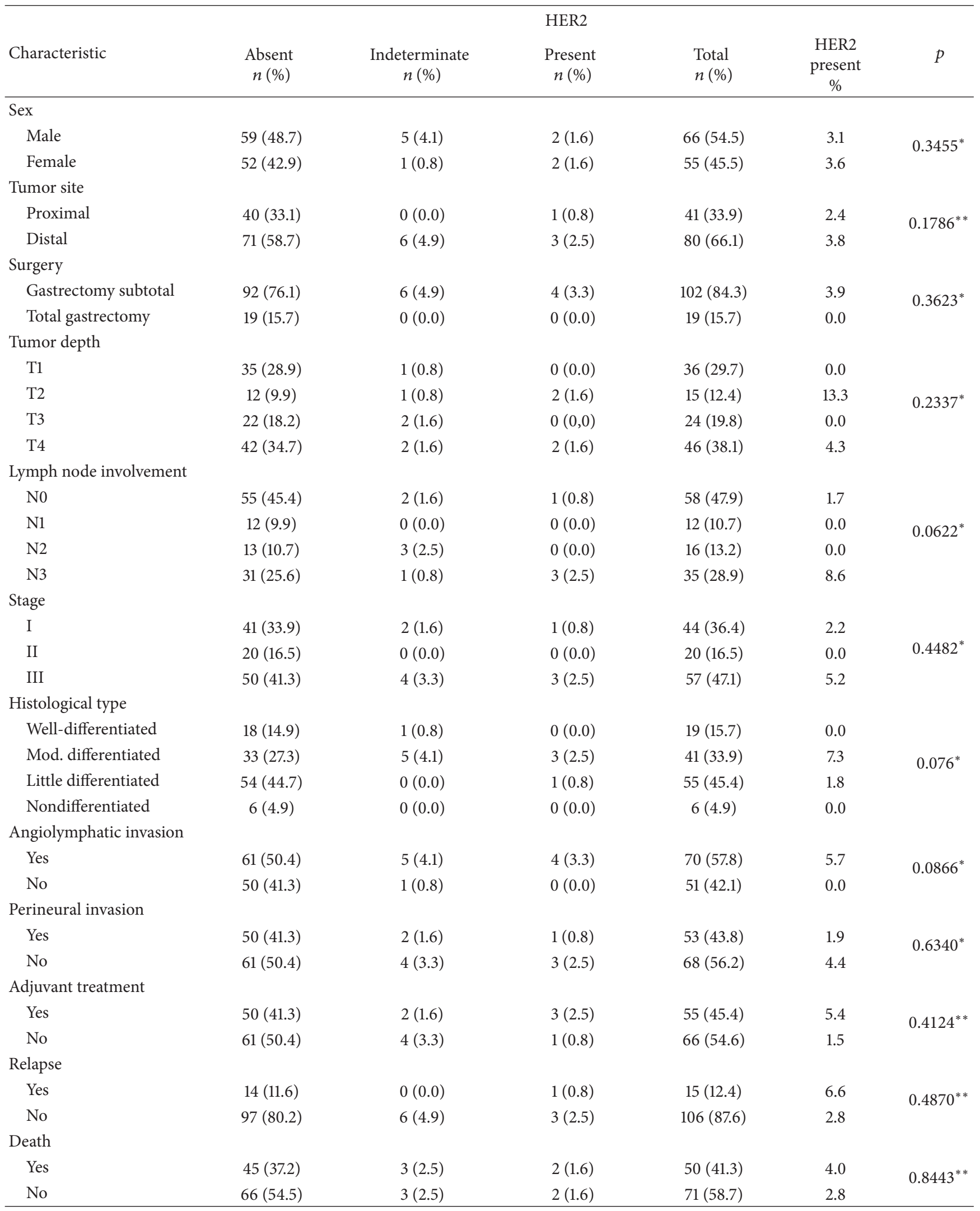

\footnotetext{
${ }^{*}$ Chi-square test.
}

${ }^{* *}$ Fisher's exact test. 
TABLE 2: Correlation of age, survival time, and lymph node involvement in patients with HER2 expression.

\begin{tabular}{|c|c|c|c|c|}
\hline \multirow{2}{*}{ Characteristic } & \multicolumn{3}{|c|}{ HER2 } & \multirow{2}{*}{$p$} \\
\hline & Absent & Indeterminate & Present & \\
\hline \multirow{6}{*}{ Age (years) } & $N=111$ & $N=6$ & $N=4$ & \multirow{6}{*}{$0.1195^{*}$} \\
\hline & $\operatorname{Min}=26$ & $\operatorname{Min}=60$ & $\operatorname{Min}=48$ & \\
\hline & $\operatorname{Max}=83$ & $\operatorname{Max}=89$ & $\operatorname{Max}=71$ & \\
\hline & Median $=61.0$ & Median $=89.5$ & Median $=56.5$ & \\
\hline & Average $=60.1$ & Average $=71.0$ & Average $=58.0$ & \\
\hline & $\mathrm{SD}=12.3$ & $\mathrm{SD}=9.8$ & $\mathrm{SD}=10.6$ & \\
\hline \multirow{6}{*}{ Survival time (months) } & $N=111$ & $N=6$ & $N=4$ & \multirow{6}{*}{$0.6679^{*}$} \\
\hline & $\operatorname{Min}=1$ & $\operatorname{Min}=1$ & $\operatorname{Min}=4$ & \\
\hline & $\operatorname{Max}=82$ & $\operatorname{Max}=60$ & $\operatorname{Max}=46$ & \\
\hline & Median $=42$ & Median $=42.5$ & Median $=42.5$ & \\
\hline & Average $=39.3$ & Average $=34.3$ & Average $=33.8$ & \\
\hline & $\mathrm{SD}=25.0$ & $\mathrm{SD}=24.7$ & $\mathrm{SD}=19.9$ & \\
\hline \multirow{6}{*}{ Positive lymph nodes } & $N=111$ & $N=6$ & $N=4$ & \multirow{6}{*}{$0.5648^{*}$} \\
\hline & $\operatorname{Min}=0$ & $\operatorname{Min}=0$ & $\operatorname{Min}=0$ & \\
\hline & $\operatorname{Max}=64$ & $\operatorname{Max}=8$ & $\operatorname{Max}=11$ & \\
\hline & Median $=1.0$ & Median $=4.0$ & Median $=8.0$ & \\
\hline & Average $=5.8$ & Average $=3.5$ & Average $=6.8$ & \\
\hline & $\mathrm{SD}=10.2$ & $\mathrm{SD}=3.1$ & $\mathrm{SD}=4.8$ & \\
\hline
\end{tabular}

${ }^{*}$ Kruskal-Wallis variance analysis.

characteristics according to the HER2 status. Among all the patients studied, $4(3.3 \%)$ were considered positive, 6 (4.9\%) indeterminate, and 111 (91.7\%) negative. There was no statistical correlation between the presence of HER2 and all studied characteristics.

\section{Discussion}

The human epithelial growth factor receptors are a family of four structurally similar homologous members (HER1 to HER4), containing an extracellular binder component, a transmembrane hydrophobic segment, and domain with tyrosine-kinase intracellular activity [20]. Its superexpression is generally associated with tumors of epithelial origin, but with much varied frequency; studies focusing on gastric cancer or esophagogastric transition found rates varying from 5 to $25 \%[6,9]$. We found positivity in $3.3 \%$, a little under the literature data. We can attribute this finding to some situations. One aspect may have been the fact that we did not perform an additional analysis of the group considered "indeterminate" (2+ reactions), through in situ hybridization tests, as some authors suggest [21]. Only for comparative purposes, if we take into account the results found by Van Cutsem et al. [22] when studying the reactions of HER2 obtained both by immunohistochemistry and by in situ hybridization, in which only approximately half of the group with $2+$ reactions were in fact positive, we might have obtained a result approaching $6 \%$, which would be in agreement with the current literature. Another situation that seems to increase the incidence of HER2+ is the proximal third tumors $[22,23]$, but such a site accounts for only onethird of the sample of our patients. Moreover, some studies
[24] suggest an association between greater expression of HER 2 and the presence of hepatic metastases. Nevertheless, no patients in this situation were included in our study, as we understand that both the metastatic disease and the residual tumor following palliative resections are situations which clearly compromise the survival time and interfere with the analysis of the results, as one of the objectives of this study was to verify the incidence of HER2 expression in gastric cancer and its possible relationship with survival. Lastly, it is well known that the incidence of HER2 seems to be higher among Japanese patients than Western patients. Even with the very well known Brazilian history of Japanese people migrating to our country over the last decades, only five patients were descendants from Japanese people, with just one positive for HER2 immunohistochemistry reaction. Due to the low number of patients, though, the statistical analysis of this characteristic may not be reliable.

As previously stated, in various published studies the intermediate results ( $2+$ reactions) were submitted to a new analysis using fluorescent in situ hybridization (FISH) or chromogenic in situ hybridization (CISH), thus obtaining only positive or negative results. In a post hoc analysis of the data obtained in the ToGA Trial [12], which studied the effects of trastuzumab associated with chemotherapy in patients with gastric and esophagogastric transition cancers which expressed HER2, Van Cutsem et al. compared the results obtained both by immunohistochemistry and by FISH [22]. The initial analysis showed a general agreement of $87.2 \%$, with 3,280 patients having been tested. For those with $3+$ reactions to the immunohistochemistry, the agreement was very high, $94.9 \%$. In the same manner, for reactions 0 and $1+$, it was $92.4 \%$ for negative FISH. However, the $2+$ reactions 
presented only $54.6 \%$ of agreement for positive FISH. For mathematical test purposes, the authors themselves excluded the $2+$ results, thus finding a new overall agreement of $92.8 \%$, which was very satisfactory. Because of some limitations in our Pathology Department, such as absence of the hybridizer instrument for in situ hybridization and lack of financial support for acquiring it and the HER2/CEN-17 probe, we could not perform the test in order to clarify the HER2undetermined $(2+)$ results. We intend to perform the FISH study in a near future. In this manner we opted not to include the 2+ results in the "positive" group without having the confirmation of their real expression, a factor at times encountered in some publications, which is of questionable methodology.

The real implication of the superexpression of HER2 as a risk factor for a worse prognosis in patients with gastric cancer has not yet been established. In the present study, there was no statistical difference as to the evolution of these patients, even though the low number of positive cases might have influenced such a result. Nonetheless, in a recent revision of meta-analyses, Nagaraja and Eslick analyzed nine large studies [25] and, of these, eight showed some type of worse evolution of HER2-positive patients. Only one revision study (11 publications, 4,569 patients), which followed the same selection methodology as the ToGA Trial, did not show a statistical relationship between the relapse-free survival and the overall survival with the expression of HER2 [11]. If we consider the few studies performed on the Brazilian population, we also found this discrepancy. Following the analysis of the relationship of the four members of the epithelial receptor family, one of the studies showed a worse evolution as for survival for those with superexpression of HER2 and HER3 [26]. This same study, however, presented other results, which were very similar to ours: a low incidence of positive HER2 (only $3 \%$ of $3+$ reactions) and a greater expression of this receptor in more differentiated histological types (they found a statistically relevant relationship, while our number of cases was close to this, with $p=0.076$ ). Conversely, another national study, which also analyzed the expression of the whole epithelial receptor family, showed a worse evolution only in the more advanced stages of the disease, without a relationship with the expression of the same receptors [27]. Finally, in a more recent publication, whose objective was to analyze the clinical-pathological aspects of patients with gastric cancer and the expression of HER2 and IMP3 (cytoplasmic receptor), there was no evidence of a worse evolution upon comparison with the superexpression of HER2 [28].

Even though our study has not shown a statistical difference between the diverse clinical-pathological characteristics and the expression of HER2, the relationship between the lymph node involvement $(p=0.0622)$ and the angiolymphatic invasion ( $p=0.0866)$ called our attention. In a recent publication, Jin et al. analyzed the clinical, pathological, and molecular characteristics of 1,104 patients with early gastric cancer submitted to gastrectomy with lymphadenectomy, showing that 104 patients $(9.4 \%)$ presented with lymph node involvement [29]. After multivariate analysis of risk factors for lymphatic dissemination, one of the characteristics found was the HER2 expression. Matsumoto et al. analyzed the HER2 expression in patients with gastric cancer and lymph node involvement submitted to neoadjuvant chemotherapy, attempting to define if there was room for trastuzumab or similar drugs in the preoperative treatment [30]. They found $27 \%$ HER2 positivity in N2+ or N3+ patients, while in the ToGA Trial, with similar methodology, the overall positivity was $12.2 \%$. This is an interesting piece of data because the lymph node involvement is known to be one of the worst factors for a bad prognosis for the patient with gastric cancer.

In this manner, in light of the similarity with other studies, we found a relatively low HER2 positivity in our gastric carcinoma cohort and we did not find a statistical relationship with the survival or with clinical characteristics. Albeit, when present, HER2 becomes one more treatment option in this disease with so few satisfactory results when in its advanced phase. Studies with new drugs are proving to be more and more promising, especially when combined with already established therapies. It is possible that one might not be able to establish an adequate relationship between prognosis and clinical characteristics specific to the superexpression of HER2, but certainly the research into its expression is very valid. As long as every viable treatment should be offered to the patient, defining if he or she presents with conditions to be submitted to a target treatment seems essential to us.

\section{Competing Interests}

The authors declare no competing interests.

\section{References}

[1] Estimativa 2016, Incidência de Câncer no Brasil/Instituto Nacional de Câncer José Alencar Gomes da Silva, INCA, Rio de Janeiro, Brazil, 2015.

[2] J. Ferlay, I. Soerjomataram, M. Ervik et al., GLOBOCAN 2012 v1.0, Cancer and Mortality Worldwide: IARC CancerBase No. 11, International Agency for Research on Cancer, Lyon, France, 2013, http://globocan.iarc.fr.

[3] A. L. Schechter, D. F. Stern, L. Vaidyanathan et al., "The neu oncogene: an erb-B-related gene encoding a 185,000-M(r) tumour antigen," Nature, vol. 312, no. 5994, pp. 513-516, 1984.

[4] H.-S. Cho, K. Mason, K. X. Ramyar et al., "Structure of the extracellular region of HER2 alone and in complex with the Herceptin Fab," Nature, vol. 421, no. 6924, pp. 756-760, 2003.

[5] X. X. He, L. Ding, Y. Lin, M. Shu, J. M. Wen, and L. Xue, "Protein expression of HER2, 3, 4 in gastric cancer: correlation with clinical features and survival," Journal of Clinical Pathology, vol. 68, no. 5, pp. 374-380, 2015.

[6] M. Yan, M. Schwaederle, D. Arguello, S. Z. Millis, Z. Gatalica, and R. Kurzrock, "HER2 expression status in diverse cancers: review of results from 37,992 patients," Cancer and Metastasis Reviews, vol. 34, no. 1, pp. 157-164, 2015.

[7] Y. Kurokawa, N. Matsuura, Y. Kimura et al., "Multicenter largescale study of prognostic impact of HER2 expression in patients with resectable gastric cancer," Gastric Cancer, vol. 18, no. 4, pp. 691-697, 2015.

[8] H. S. Song, Y. R. Do, I. H. Kim, S. S. Sohn, and K. Y. Kwon, "Prognostic significance of immunohistochemical expression of EGFR and c-erbB-2 oncoprotein in curatively resected gastric 
cancer," Cancer Research and Treatment, vol. 36, no. 4, pp. 240245, 2004.

[9] A. F. C. Okines and D. Cunningham, “Trastuzumab: a novel standard option for patients with HER-2-positive advanced gastric or gastro-oesophageal junction cancer," Therapeutic Advances in Gastroenterology, vol. 5, no. 5, pp. 301-318, 2012.

[10] M. Terashima, K. Kitada, A. Ochiai et al., "Impact of expression of human epidermal growth factor receptors EGFR and ERBB2 on survival in stage II/III gastric cancer," Clinical Cancer Research, vol. 18, no. 21, pp. 5992-6000, 2012.

[11] J. Gu, L. Zheng, Y. Wang, M. Zhu, Q. Wang, and X. Li, "Prognostic significance of HER2 expression based on trastuzumab for gastric cancer (ToGA) criteria in gastric cancer: an updated meta-analysis," Tumor Biology, vol. 35, no. 6, pp. 5315-5321, 2014.

[12] Y.-J. Bang, E. Van Cutsem, A. Feyereislova et al., "Trastuzumab in combination with chemotherapy versus chemotherapy alone for treatment of HER2-positive advanced gastric or gastrooesophageal junction cancer (ToGA): a phase 3, open-label, randomised controlled trial," The Lancet, vol. 376, no. 9742, pp. 687-697, 2010.

[13] A. Okines, D. Cunningham, and I. Chau, "Targeting the human EGFR family in esophagogastric cancer," Nature Reviews Clinical Oncology, vol. 8, no. 8, pp. 492-503, 2011.

[14] E. C. Smyth and D. Cunningham, "Targeted therapy for gastric cancer," Current Treatment Options in Oncology, vol. 13, no. 3, pp. 377-389, 2012.

[15] S. Iqbal, B. Goldman, C. M. Fenoglio-Preiser et al., "Southwest Oncology Group study S0413: a phase II trial of lapatinib (GW572016) as first-line therapy in patients with advanced or metastatic gastric cancer," Annals of Oncology, vol. 22, no. 12, Article ID mdr021, pp. 2610-2615, 2011.

[16] J. R. Hecht, Y. J. Bang, S. Qin et al., "Lapatinib in combination with capecitabine plus oxaliplatin (CapeOx) in HER2-positive advanced or metastatic gastric, esophageal, or gastroesophageal adenocarcinoma (AC): the TRIO-013/LOGiC Trial," Journal of Clinical Oncology, vol. 31, supplement 1, abstract LBA4001, 2013.

[17] T. Satoh, R.-H. Xu, H. C. Chung et al., "Lapatinib plus paclitaxel versus paclitaxel alone in the second-line treatment of HER2-amplified advanced gastric cancer in Asian populations: TyTAN-a randomized, phase III study," Journal of Clinical Oncology, vol. 32, no. 19, pp. 2039-2049, 2014.

[18] M. Hofmann, O. Stoss, D. Shi et al., "Assessment of a HER2 scoring system for gastric cancer: results from a validation study," Histopathology, vol. 52, no. 7, pp. 797-805, 2008.

[19] T. Sano and Y. Kodera, "Japanese classification of gastric carcinoma: 3rd English edition," Gastric Cancer, vol. 14, no. 2, pp. 101-112, 2011.

[20] T. Akiyama, C. Sudo, H. Ogawara, K. Toyoshima, and T. Yamamoto, "The product of the human c-erbB-2 gene: a 185kilodalton glycoprotein with tyrosine kinase activity," Science, vol. 232, no. 4758, pp. 1644-1646, 1986.

[21] Y. K. Wang, C. F. Gao, T. Yun et al., "Assessment of ERBB2 and EGFR gene amplification and protein expression in gastric carcinoma by immunohistochemistry and fluorescence in situ hybridization," Molecular Cytogenetics, vol. 4, no. 1, article no. $14,2011$.

[22] E. Van Cutsem, Y.-J. Bang, F. Feng-yi et al., "HER2 screening data from ToGA: targeting HER2 in gastric and gastroesophageal junction cancer," Gastric Cancer, vol. 18, no. 3, pp. 476-484, 2014.

[23] H. Otsu, E. Oki, A. Ikawa-Yoshida et al., "Correlation of HER2 expression with clinicopathological characteristics and prognosis in resectable gastric cancer," Anticancer Research, vol. 35, no. 4, pp. 2441-2446, 2015.

[24] T. Saito, H. Nakanishi, Y. Mochizuki et al., "Preferential HER2 expression in liver metastases and EGFR expression in peritoneal metastases in patients with advanced gastric cancer," Gastric Cancer, vol. 18, no. 4, pp. 711-719, 2015.

[25] V. Nagaraja and G. D. Eslick, "HER2 expression in gastric and oesophageal cancer: a meta-analytic review," Journal of Gastrointestinal Oncology, vol. 6, no. 2, pp. 143-154, 2015.

[26] M. D. Begnami, E. Fukuda, J. H. T. G. Fregnani et al., "Prognostic implications of altered human epidermal growth factor receptors (HERs) in gastric carcinomas: HER2 and HER3 are predictors of poor outcome," Journal of Clinical Oncology, vol. 29, no. 22, pp. 3030-3036, 2011.

[27] A. A. A. Jácome, D. R. Wohnrath, C. Scapulatempo Neto et al., "Prognostic value of epidermal growth factor receptors in gastric cancer: a survival analysis by Weibull model incorporating long-term survivors," Gastric Cancer, vol. 17, no. 1, pp. 76-86, 2014.

[28] E. A. M. Damasceno, F. P. Carneiro, A. V. de Magalhães et al., "IMP3 expression in gastric cancer: association with clinicopathological features and HER2 status," Journal of Cancer Research and Clinical Oncology, vol. 140, no. 12, pp. 2163-2168, 2014.

[29] E. H. Jin, D. H. Lee, S.-A. Jung et al., "Clinicopathologic factors and molecular markers related to lymph node metastasis in early gastric cancer," World Journal of Gastroenterology, vol. 21, no. 2, pp. 563-570, 2015.

[30] T. Matsumoto, M. Sasako, J. Mizusawa et al., "HER2 expression in locally advanced gastric cancer with extensive lymph node (bulky N2 or paraaortic) metastasis (JCOG1005-A trial)," Gastric Cancer, vol. 18, no. 3, pp. 467-475, 2015. 

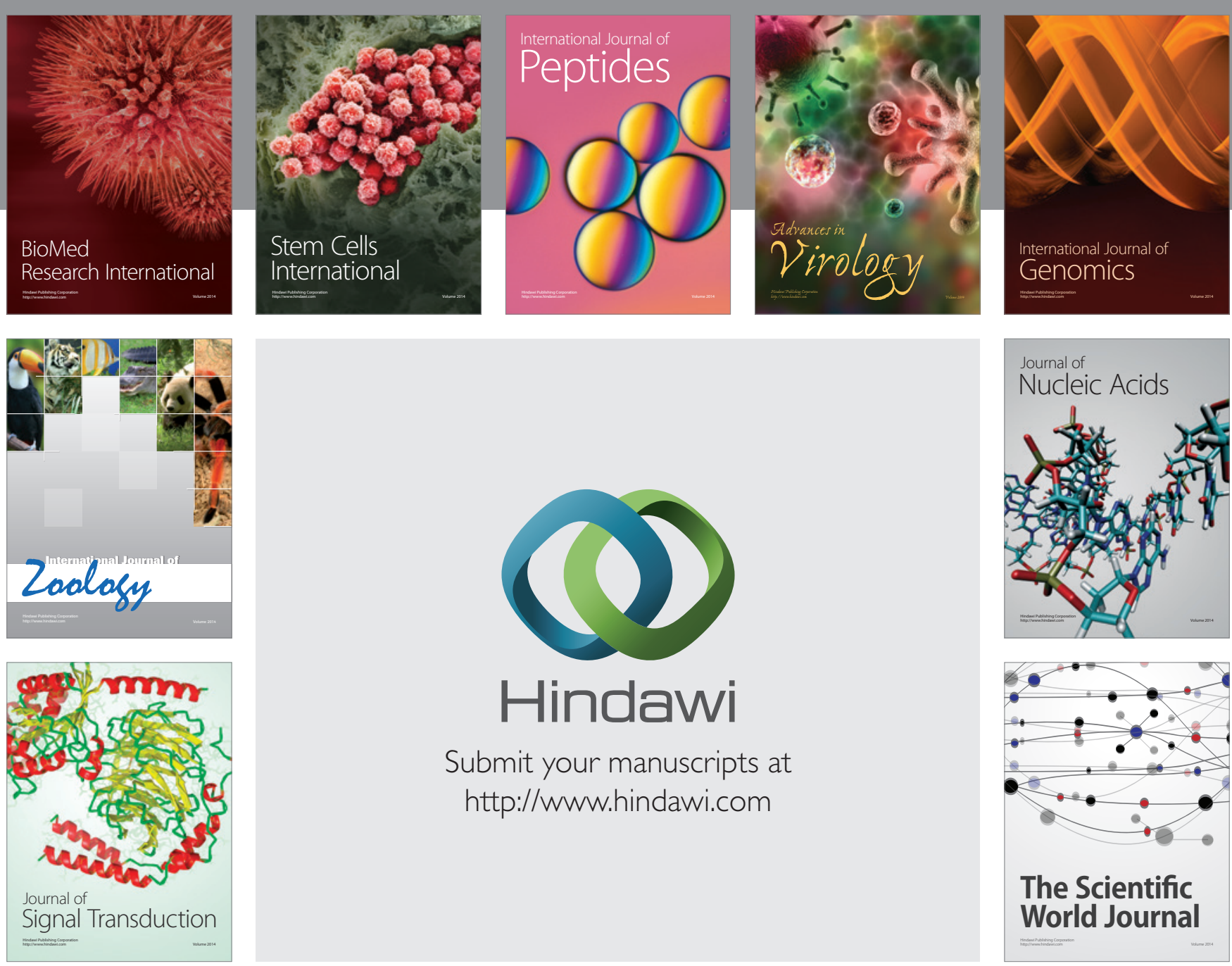

Submit your manuscripts at

http://www.hindawi.com
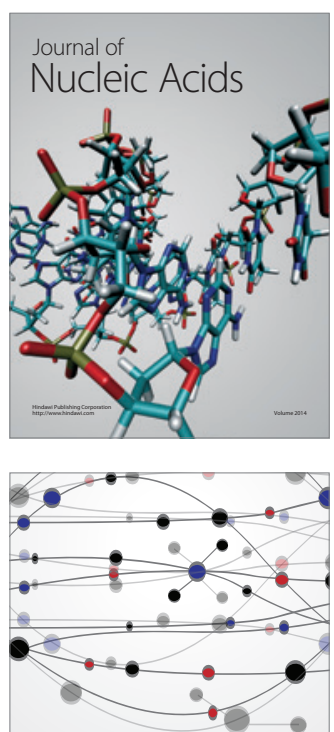

The Scientific World Journal
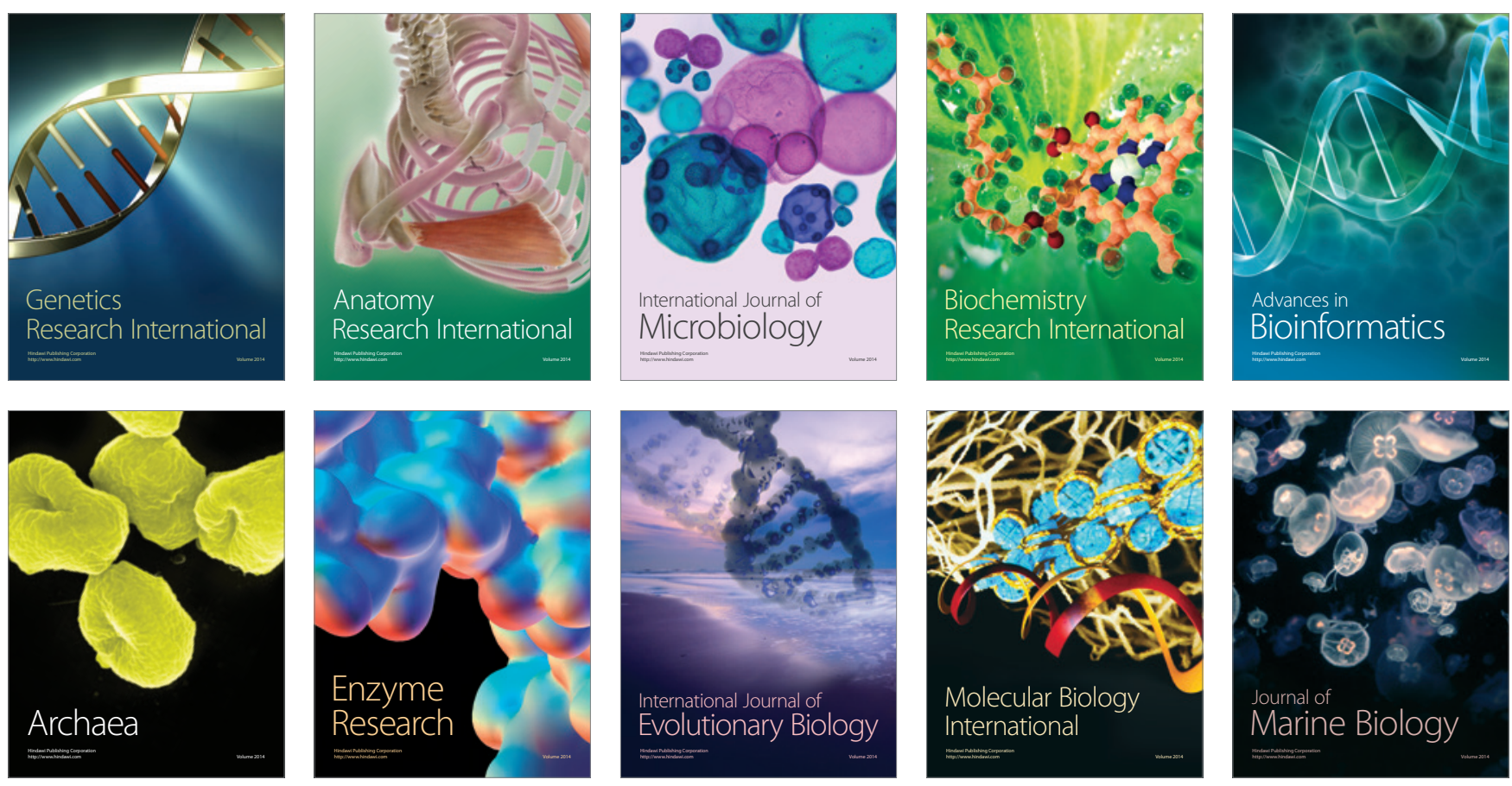